EOMmUn: Communication et organisation

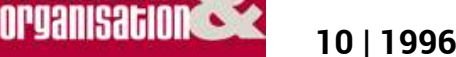

La recherche en communication

\title{
De la méthode
}

\section{Paul Stryckman}

\section{OpenEdition}

Journals

Édition électronique

URL : http://journals.openedition.org/communicationorganisation/1867

DOI : 10.4000/communicationorganisation. 1867

ISSN : 1775-3546

\section{Éditeur}

Presses universitaires de Bordeaux

\section{Édition imprimée}

Date de publication : 1 novembre 1996

ISSN : 1168-5549

\section{Référence électronique}

Paul Stryckman, « De la méthode », Communication et organisation [En ligne], 10 | 1996, mis en ligne le 26 mars 2012, consulté le 19 avril 2019. URL : http://journals.openedition.org/ communicationorganisation/1867 ; DOI : 10.4000/communicationorganisation.1867

Ce document a été généré automatiquement le 19 avril 2019

(c) Presses universitaires de Bordeaux 


\section{De la méthode}

\section{Paul Stryckman}

1 Le langage courant confond méthode et méthodologie au point que dans bien des textes le terme de méthodologie remplace la simple description de la méthode ou des méthodes. C'est bien ainsi que la presse parle de la «méthodologie du sondage » et limite cette dernière à l'identité des commanditaires, à l'échantillonnage, aux dates de la cueillette téléphonique. Il saute aux yeux que la « méthode » d'hier est devenue la «méthodologie » d'aujourd'hui, tout comme les problèmes sociaux ou techniques d'autrefois se font maintenant des problèmes "sociologiques" ou «technologiques». La réalité des comportements et des choses serait, semblerait moins consistante, voire valorisante, que la réflexion sur ces réalités! On peut parler d'un glissement métonymique puisqu'il semblerait bien y avoir un rapport entre méthodologie et méthode. Ce glissement dans nos préférences lexicales renvoie à la question bien délicate des traitements rhétoriques dans le langage des sciences humaines et, à moyen terme, de leurs effets de contamination dans le langage ordinaire.

Dans le présent article, nous proposons de réfléchir sur notre lexique méthodologique afin d'y apporter un éclairage sémantique susceptible de mettre en question le traitement que nous lui réservons. Prenons, comme point de départ, une remarque d'un classique qui ouvre sur le vif du sujet.

«Ainsi mon dessein n'est pas d'enseigner ici la méthode que chacun doit suivre pour bien conduire sa raison, mais seulement de faire voir en quelle sorte j'ai tâché de conduire la mienne. » (Descartes, I, pp. 60-64) [italiques par nous].

La citation est exemplaire parce qu'elle révèle clairement le va-et-vient sur un double registre. D'une part, le « faire voir en quelle sorte » de la conduite indique bien l'analyse d'un cheminement, d'autre part, le «que chacun doit suivre » évoque une norme, une règle à donner au cheminement. La méthode concerne donc le chemin suivi ou le chemin à suivre. Le terme méthode, composé des termes grecs meta-odos, peut être traduit par « chemin suivi ». Si ce "chemin » offre des garanties de savoir-faire, il devient aussi un chemin à suivre, un passage obligé à respecter. Le terme méthode reçoit ainsi deux acceptions bien différentes. Dans son acception descriptive, la méthode expose le chemin suivi, chemin par lequel un certain résultat est atteint sans que ce chemin ait été fixé 
d'avance. Dans son acception normative, la méthode présente le chemin à suivre, elle expose les règles validant la démarche.

\section{Deux méthodologies disponibles}

4 La méthodologie, méth-odo-logie [meta-odos-logos], devrait être la réflexion sur le chemin suivi, la réflexion sur la poursuite du travail. La méthodologie au sens strict du terme devient ainsi la réflexion correspondant nécessairement à chacune de ces deux acceptions retenues. Elle est réflexion positive sur le chemin-suivi ou normative sur le chemin-à-suivre. Ignorer cette distinction est un manque de méthode !

5 La méthodologie positive examine de façon critique le chemin suivi. Elle peut encore le faire de deux façons différentes : durant la recherche en cours ou une fois la recherche terminée.

6 Dans la méthodologie du "chemin faisant ", le chercheur doit nécessairement prendre distance et se regarder "cherchant» ou s'interroger sur son savoir-faire devant les obstacles rencontrés. Dans ce sens, la réflexion méthodologique consiste principalement à prendre conscience des choix pris durant l'avancement de la recherche. «La méthode comme objet, dit R. Ledrut, n'est ni avant ni après! Toujours présente, elle est en rapport permanent d'action réciproque avec l'objet (et le sens) de la Science. La méthode naît à chaque instant de la réflexion sur la spontanéité du mouvement de recherche » (1981, p. 216.) On devrait dire que cette pratique est thématiquement irrécupérable puisqu'elle porte sur mille et une décisions ou mises au point inhérentes à toute recherche in actu.

7 La méthodologie positive peut aussi se faire une fois la recherche accomplie pour rendre compte des difficultés rencontrées ou pour établir le bilan des choix effectués durant la recherche. Cette réflexion sur le fait accompli, cette méthodologie post factum semble la seule possible auprès de certains auteurs. C'est l'optique prise par E. Morin quand il affirme :
«À l'origine, le mot méthode signifiait cheminement. Ici, il faut accepter de cheminer sans chemin, de faire le chemin dans le cheminement. Ce que disait Machado : Caminante no hay camino, se hace camino al andar. La méthode ne peut se former que pendant la recherche; elle peut se dégager et ne se formuler qu'après, au moment où le terme redevient un nouveau point de départ, cette fois doté de méthode » $(1977$, p. 22.)

La méthodologie positive en tant que réflexion explicite, en tant que texte dans un rapport de recherche par exemple, peut donc se présenter sous deux formes. Elle peut être une réflexion d'accompagnement dans les différentes étapes de la recherche. Ce serait alors un journal de bord rendant compte du cheminement et des décisions prises. Elle peut aussi être une réflexion de synthèse, une récapitulation générale de la démarche suivie. Comme nous pouvons le constater, dans la grande majorité des publications sur des recherches en communication, les paragraphes sur la méthodologie se limitent à une très rapide description-bilan des techniques appliquées. Dans ce sens, il faut reconnaître que la méthodologie ou plutôt, si nous devons accepter le glissement métonymique relevé, la pensée méthodologique n'est pas très développée dans la présentation des travaux en communication. Il y a description des techniques utilisées sans grande exposition des options possibles ni des choix fixés.

9 La méthodologie normative traite de ce qu'il faut faire, du chemin qu'il faut suivre. Comme nous le verrons en fin d'article, cette dernière est explicitement fournie dans les 
manuels de méthodologie. Disons toutefois que sa principale caractéristique réside dans son affirmation d'une définition d'une science normale, laquelle détermine une méthode correspondante. Par science normale, il faut entendre les pratiques respectant la méthodologie exposée dans les manuels du savoir-faire qui font autorité (Kuhn, 1972). Dans la mesure où celle-ci est confondue avec un ensemble de techniques à appliquer, la méthodologie normative se réduit à un ensemble de recettes garantissant un savoir-faire " scientifique " sans aucune mise en question des procédures ni des objectifs à atteindre. Ce type de réflexion présente l'inconvénient de se préoccuper principalement d'applications expérimentales sans analyser les contraintes déjà inscrites dans la formulation du questionnement, voire celles inhérentes aux langages qui caractérisent le passage du théorique à l'empirique.

On peut donc avancer que toute absence de méthodologie positive dans la présentation des résultats d'une recherche, c'est-à-dire tout manque d'explicitation de la méthode suivie, repose sur une présomption tacite d'une méthodologie normative donnée. Cette présomption pose sans examen critique la scientificité de la problématique et de son objet ou de son construit sur la seule base de techniques rapidement exposées ${ }^{1}$.

\section{Une méthode ou des méthodes?}

\section{Connaissances et pratiques}

Mais alors, faut-il parler de méthodes ou de la méthode? La question exige une clarification complémentaire. On peut parler de la méthode d'une recherche, et le terme reste au singulier, pour tracer le chemin-à-suivre dans la réalisation d'une recherche. Le terme est alors purement générique et il équivaut à travail de recherche. On peut aussi parler de la méthode en sous-entendant la méthode expérimentale en tant que garantie d'une connaissance "scientifique ». Le terme reçoit alors une acception très particulière parce que la méthode expérimentale suppose un certain nombre de conditions épistémologiques pour cerner l'objet de recherche, l'observer, et de contraintes logiques validant la démarche à suivre dans la preuve. En d'autres mots, il s'agit essentiellement de confronter [les conditions épistémologiques] une idée, une hypothèse à une expérience articulant une observation susceptible [contraintes logiques] de mettre cette idée, cette hypothèse à l'épreuve d'une expérience empirique. C'est dire que la raison expérimentale est devenue non seulement un chemin vers une "réalisation ${ }^{2}$ " en imposant des procédés éprouvés de confirmation empirique, mais elle est aussi la norme fondatrice qui, elle seule, prétend donner raison au réel qu'elle construit. Il est aujourd'hui admis que les conditions et contraintes mentionnées appartiennent à la « raison expérimentale» de la science moderne (Ledrut, 1981, P - 17; Piaget, 1971, pp. 38-42.)

12 Il va sans dire que cette raison expérimentale privilégie un mode de production dite scientifique essentiellement articulée sur une méthode de travail. En effet, dans le paradigme de la science expérimentale, l'objet devient un être scientifique dans la mesure où il est défini par une relation observable et "répétable », c'est-à-dire par une relation qui peut être enregistrée par des mesures.

«Mais la méthode même de la science est de rechercher de telles relations

« répétables » et, à travers elles, d'atteindre et de désigner des êtres scientifiques qui

constitueront les objets. » (Ullmo, 1969, p. 27.) 
13 Ceci veut dire que le scientifique se méfie du concret, de l'empirique parce qu'il risque de n'en faire qu'une lecture intuitive. En pensant en termes de relation, le scientifique va "déréifier » et "déconcrétiser » les qualités apparentes de l'objet-phénomène pour réaliser et montrer des êtres relationnels qui échappent souvent à l'expérience immédiate. On peut comprendre que la systématique de l'opération impose un cheminement, une méthode donnant accès à l'intelligence des "choses ». Ceci suppose nécessairement que le sens n'est pas donné et qu'il faudra élaborer des stratégies et appliquer des techniques pour produire une connaissance dite empirique.

Mais la méthode - réduite implicitement à la méthode expérimentale - reste encore un terme générique. Pourquoi ? Parce que les conditions épistémologiques de l'observation empirique et les contraintes logiques de la preuve instaurent nécessairement une série de choix assurant le passage de l'abstrait au concret. Ici, il existe un large éventail de possibilités pour passer des concepts et des hypothèses théoriques [abstraites] aux hypothèses opératoires, c'est-à-dire observables, et aux variables, mesures appliquées à ces observations. Certains auteurs parlent de méthodes des sciences humaines, par exemple, pour discerner les cheminements propres à la psychologie, la science politique, la sociologie et autres disciplines expérimentales. Nous avons alors affaire à des méthodes expérimentales spécifiques à un champ d'étude.

15 Il reste à lever l'ambiguïté de la référence à l'expérience en tant que fondement de la connaissance. En effet, le développement des pratiques ne permet pas de confondre « raison expérimentale » et traitement empirique. Du point de vue de la connaissance, les rapports avec l'empirique déterminent des pratiques multiples que la réflexion méthodologique ne peut pas ignorer sous l'impérialisme du scientisme expérimental ${ }^{3}$. Ainsi une approche peut paraître plus empirique en laissant plus de place au phénomène par le seul fait qu'elle part plus humblement d'expériences immédiates dites cliniques. Terme originaire de la pratique médicale, la clinique désigne l'observation « auprès du lit du malade " [gr. klinicos = lit] par opposition à celle conduite sur fond théorique ou médiatisée par un protocole d'enregistrement de données. L'expérience clinique procure ainsi un savoir tiré de la pratique immédiate. Dans l'expérience clinique, une vérité s'instaure dans la pratique, dans l'expérience d'une totalité (la personne du patient « passe » avant sa maladie) par opposition à la théorie ou à la réflexion spéculative. Elle laisse place à des sujets acteurs, des essais, des interventions, des approches multiformes convergeant vers une compréhension globale. Il va sans dire que les méthodes cliniques, toujours proches des faits, des phénomènes, opèrent dans des contextes épistémologiques très différents de l'approche expérimentale. De nombreuses controverses suscitées par les méthodes cliniques tournent autour du sens donné à la pratique ou à son analyse dans le développement des connaissances (Berg, Smith, 1985).

À l'examen des développements des méthodes en sciences humaines, l'analyse des pratiques des dernières décennies a soulevé des débats fort instructifs du point de vue du statut des méthodes. Nous voulons en présenter deux. Le premier débat concerne la pratique de la science comme pratique inscrite dans un contexte de valeurs, de priorités marqué par des moments culturels et des orientations socio-politiques ${ }^{4}$. Son analyse appartient plus à la sociologie de la science qu'à une question de méthode. Nous l'appliquons à la notion de méthodologie féministe. D'autre part, la pratique même de la recherche dite scientifique n'est pas sans poser de sérieuses interrogations sur les canons de sa méthodologie au point de mettre en question la pertinence même de la méthode expérimentale. Ce second débat examine les conditions épistémologiques que la méthode 
expérimentale impose. C'est l'interrogation qu'entretient gravement l'ethnométhodologie.

\section{Peut-on parler d'une méthodologie féministe?}

17 Un bel exemple du contexte socio-politique de la recherche est certainement le cas du développement de recherches dites féministes. Il suffit de parcourir la moindre bibliographie sur le sujet ou les programmes d'études universitaires pour se rendre compte que ce sont des sujets de recherche ou d'étude relativement récents. Ce sont des contextes culturels et des actions sociales qui ont généré les conditions de possibilité de poser les problèmes soulevés par ces recherches, de « réaliser » leur objet de recherche. Si ces recherches sont récentes, il faut admettre que les questions à poser ou à résoudre n'ont pu l'être qu'à partir de situations données et d'un discours toléré.

18 Examinons cette dernière remarque dans l'analyse de deux situations empiriques fort différentes. La situation I serait celle des femmes travaillant en relations publiques. Une analyse historique constate que, jusqu'à tout récemment, il y avait peu de recherches sur les femmes dans les métiers de la communication publique dans un pays donné ${ }^{5}$. La situation IIest tirée du domaine de la gérontologie sociale. Une recherche documentaire peut montrer qu'il y a bien plus de publications et de travaux sur le vieillissement des hommes que sur les femmes ${ }^{6}$ et ce malgré le poids démographique des femmes âgées, poids mis en évidence depuis belle lurette par tant de démographes masculins. Les situations I et II permettent de tirer plusieurs inférences sur les «conditions de possibilité » de poser un objet de recherche, sur les valeurs de ces scientifiques, sur les contextes organisationnels qui ont subventionné ces recherches. Examinons rapidement quelques-unes de celles-ci.

Première constatation. Les chercheurs sont masculins et la réalité observée est masculine. Ceci peut laisser croire qu'il se produit une réduction de vision, voire une «scotomisation" au point que ces chercheurs masculins ne voient que des problèmes affectant des hommes. Cette lecture serait acceptable pour la situation I, le nombre de femmes concernées étant quantitativement négligeable. C'est dire que l'objet de recherche ne pourrait être que « masculin ». On pourrait admettre que, dans la situation I, la présence des femmes dans les pratiques des relations publiques étant " objectivement », c'est-à-dire numériquement, négligeable, on se trouve devant un objet de recherche réduit, sans pouvoir conclure qu'il y a vraiment une réduction de l'objet produite par les chercheurs masculins ${ }^{7}$. Cette sélection réductrice est beaucoup plus difficilement acceptable dans la situation II de la démographie sociale. Nous y reviendrons. Ceci dit, il faut toutefois reconnaître que le non-intérêt pour les cas rares ou faire appel au principe de la «négligeabilité » soutiennent un argument d'exclusion qui n'a aucune légitimité scientifique. Ce type d'argument appartient à un régime de valeurs qui ne s'interdit pas de poser une problématique critique sur le fait même que très peu de femmes font carrière en relations publiques. Ceci constitue une question méthodologique par excellence, bien préalable à toute application d'une technique de recherche sur le terrain.

20 Deuxième constatation: les femmes ne sont pas porteuses de faits ni de situations qui méritent discours et recherche. Dans cet ordre des idées, on peut avancer que la grande première césure du vieillissement peut paraître dans la mise à la retraite en tant que retrait du marché du travail, retrait qui peut être vécu [inconsciemment] comme une 
préoccupation, une angoisse auprès de gérontologues masculins au détriment d'autres césures du vieillissement vécues par les femmes, par conséquent, césures sans intérêt... parce que vécues par des femmes. Dans ce bilan d'une gérontologie masculinisée, il n'y a plus objet réduit, mais réduction de la problématique du vieillissement. On peut donc conclure que la situation II est le résultat d'une définition normée d'une réalité réduite à des problématiques typiquement masculines, formulée dans un discours spécifiquement masculin ${ }^{8}$.

On doit comprendre que les situations I et II sont de deux ordres complètement différents et que l'absence de recherches sur les femmes renvoie à des réalités objectives et des perspectives analytiques à ne pas confondre. Faut-il parler d'erreur de méthode ? Selon nous, il semble plus pertinent de distinguer ici la méthode de toute stratégie de recherche appartenant à une praxis donnée, à une problématique sélective (situation I) ou réductrice (situation II). Dans la mesure où la problématique peut être définie comme la mise en ordre d'une vision et d'un moment créateur, on ne peut plus avancer qu'il existe une ou des méthodes féministes, voire une "épistemologie féministe " (Durant, 1991), mais que sa formulation et ses choix sur l'important, le négligeable lui sont propres, la définissent sans pouvoir en déduire une "méthode " spécifique. Notons bien que nous rendons compte de la différence entre les deux situations en termes de problématique et non en termes de méthode. De ce fait, la question est clairement préalable à tout cheminement à suivre, c'est-à-dire antérieure à toute méthode. Ceci signifie que poser un problème est de l'ordre de l'invention et du questionnement. C'est une affaire de sémantique et une question éminemment théorique.

\section{Le dossier de l'ethnométhodologie}

La démarche ethnométhodologique pourrait être qualifiée de contre-scientifique dans le sens qu'elle trouve son origine dans une reformulation épistémologique assez radicale privilégiant les pratiques et les jugements du sens commun. Dans la mesure où ces pratiques et ces jugements gèrent les règles d'action et les échanges entre acteurs, on peut se demander s'ils ne sont à l'œuvre au sein de la démarche scientifique même. Ceci revient à dire que, à la limite, la démarche rationnelle revendiquée pour produire de la "science", du "scientifique " n'est pas étrangère à, voire ne peut pas exclure la rationalité avec laquelle les gens ordinaires opèrent dans leur vie quotidienne. Mais admettre ce principe, c'est ne plus classer cette rationalité comme une catégorie résiduelle et négligeable, c'est se demander si les interactions entre les membres de groupes de professionnels ou de scientifiques sont semblables à celles entre les membres de groupes profanes ${ }^{9}$. Établir une telle équation revient à s'interroger sur la spécificité de la pensée scientifique. Dans un telle mise en question, on peut aller jusqu'à se demander si toute une pratique quotidienne ne serait pas déformée au travers de la grille de la description scientifique! C'est oser insinuer que, de par sa méthode, le scientifique classique construit un réel, prétend mettre à jour des règles de pratiques sociales filtrées par sa méthode de poser le problème et de confirmer sa vérité.

Le radicalisme méthodologique de cette pensée est particulièrement critique sur le statut du langage dans la description-observation d'actions sociales, comme des échanges d'informations sur le respect [ou le non-respect] de règles. En se mettant au ras du sol de l'action sociale, l'ethnométhodologue s'interroge sur les fonctions du langage verbal non seulement dans les échanges d'informations, ciblés comme objets de recherche, mais aussi dans leur description. Peut-il y avoir une description transparente de cette 
pratique? Le langage de la description peut-il rendre compte du langage vécu dans la dynamique des échanges ciblés? En somme, de telles interrogations portent atteinte à la croyance dans la neutralité du langage descriptif comme langage « représentatif', c'est-àdire comme image verbale valide de l'action. Il est bien évident que la mise en doute de la représentativité du langage descriptif est variable selon la nature des règles à respecter sur lesquelles portent les échanges. Mais cette simple affirmation est porteuse d'une sérieuse conclusion : le langage descriptif est toujours un langage contextuel dans le sens qu'il ne dispose pas d'une potentialité virtuellement neutre. Non, le langage de la description est nécessairement un langage d'action puisqu'il ne peut pas échapper aux règles régissant « le dire et le décrire » en tant que lui-même échange d'informations... Se disant cela, l'ethnométhodologue doit renoncer à voir dans l'observation objective d'une action humaine un moment neutre pour admettre que son observation s'inscrit dans l'action même et que par conséquent toutes les deux, observation et intervention, sont imbriquées dans un même contexte de sens commun. En d'autres termes, l'ethnométhodologie ébranle sérieusement le socle de la raison expérimentale et la mise en place de ses protocoles.

\section{La notion de protocole}

Dans le contexte épistémologique de l'approche fondée sur la raison expérimentale, beaucoup d'auteurs parlent de méthodes au heu de la méthode. Pourquoi ? Parce que la raison expérimentale implique nécessairement des expérimentations précises et rigoureuses qui ne peuvent être entreprises sans de nombreuses tentatives de réalisation ou une pluralité de cheminements et d'interprétations multiples. Les méthodes ne seraient rien d'autre que des démarches particulières nécessaires à l'adaptation aux contraintes imposées par la définition de l'objet et de son traitement dans une recherche empirique.

Mais attention! Ces démarches particulières peuvent encore correspondre, selon les auteurs, à deux acceptions fort différentes. Certains utilisent le terme pour identifier des orientations générales dans la façon de cerner théoriquement et empiriquement un même objet de recherche. Ainsi, par exemple, une recherche sur une image publique résultant d'une campagne publicitaire peut opter pour des méthodes qualitatives, quantitatives, structurales.

Pour clarifier les niveaux d'analyse et éviter la confusion terminologique, il vaut mieux abandonner ici le concept de méthode et passer par le détour du terme de protocole devenu de mode dans certains milieux scientifiques. Que signifie protocole? Selon l'étymologie grecque, le protocole, c'est «ce qui est collé en premier» pour rendre authentique, pour confirmer le document ou indiquer la lecture à en faire. De là, l'idée de liste de conventions appliquées dans une opération, de mode d'emploi d'un outil, de déroulement des étapes à suivre dans une opération pour obtenir un résultat précis et reconnu valable. Le protocole est un ensemble de règles appliquées systématiquement dans l'enregistrement d'informations et dans leur traitement ultérieur. Plus un projet de recherche est complexe, c'est-à-dire plus il y a d'étapes à suivre dans sa réalisation, plus celui-ci exige différents protocoles. En nous en tenant à l'étymologie, le protocole devient donc le «texte » qui accompagne les résultats obtenus, texte permettant d'interpréter ceux-ci et d'évaluer leur validité. 


\section{Trois applications}

\section{L'opinion des organisations}

Dans un projet de recherche, on veut savoir comment des organisations expriment des opinions. La question ne manque pas d'être originale puisqu'il s'agit de savoir comment un collectif comme une organisation produit des opinions, une opinion. Le passage du pluriel au singulier mérite déjà toute une réflexion théorique ! En s'intéressant à l'idée de «l'opinion d'une organisation ", on peut se laisser prendre par le syntagme " expression d'opinion» et penser en termes d'un sondage pouvant recueillir des opinions dans l'organisation. Dans ce cas, le technique prend les devants sur la méthode. On pense immédiatement « entrevue » et enregistrement d'opinions.

Mais on peut aussi voir l'organisation comme une entité disposant d'un ou de processus de représentation ou de diffusion d'une opinion autorisée, laquelle ne serait pas nécessairement représentative des opinions des membres. Dans ce cas, l'opinion autorisée serait exprimée dans des documents, des avis officiels, des mises au point de service ou des journaux internes, des journaux officiels, des communiqués ou des conférences de presse... Mais alors, la technique se fait analyse de contenu ou analyse de discours.

On peut même pousser l'analyse plus loin et dégager, selon différentes requêtes d'information, le réseau interne de l'organisation qui permet de rejoindre le porte-parole autorisé $^{10}$. Il va sans dire que le terme même d'organisation n'est pas sans exiger alors des précisions. Car, il y a organisation et organisation. Un hôpital, une prison, une entreprise de presse, une université, une banque, une paroisse sont des collectifs qui peuvent être considérés comme des organisations présentant des structures et des objectifs très spécifiques. La question de départ doit prendre un sens en commençant par définir le type d'organisation auquel la question est appliquée et le mode ou les modes d'expression des opinions ou de l'opinion. Sans une telle clarification théorique, la recherche s'en tiendrait à construire des scénarios sans référence à des expériences organisationnelles. Sur la base d'une réflexion théorique s'inspirant du domaine de la communication dans les organisations, le travail théorique doit nécessairement élaborer de tels scénarios et les paramètres organisationnels déterminant leur mise en action respective.

Organisations, modes d'expression, opinions ou opinion sont ici des notions fondamentales qu'il faudra clarifier. Car la «raison expérimentale » exigera tôt ou tard que scénarios et paramètres soient interprétés dans des organisations concrètes ou, ce qui revient au même, que des acteurs réels, des textes, des discours, des processus d'intervention puissent être considérés comme des correspondances empiriques valides des "acteurs", des "actes de parole» définis dans la recherche théorique. Plusieurs approches sont alors possibles selon les critères pertinents aux questions posées. Une approche statique se limitera à analyser les « actes de parole » qui seront recueillis sous forme d'expressions verbales ou de textes. Une approche dynamique analysera, dans un événement passé ou en provoquant "expérimentalement» une demande d'opinion, comment et par qui des «actes de parole » émanent d'organisations. Plusieurs chemins peuvent être suivis. L'exigence méthodologique consiste ici à évaluer la pertinence et la fiabilité du chemin suivi selon un protocole donné ou à suivre selon la question posée ou l'hypothèse formulée. Jusqu'à présent la recherche conjuguera donc réflexion théorique 
et validation méthodologique sans qu'il soit question de techniques d'observation et d'analyse.

\section{La féminisation dans la production télévisuelle}

31 Des « chercheuses » constatent la faible représentativité des femmes dans la réalisation d'émissions de télévision ${ }^{11}$. Devant ce constat, elles s'interrogent sur l'influence que les réalisatrices d'émissions de télévision exercent sur le métier et sur les images diffusées par leurs émissions.

La double question posée constitue un énorme programme de recherche. L'examen de l'influence de la féminisation du métier peut se faire sous différentes approches. Il peut s'agir d'établir un bilan ponctuel, une description sociographique de la "féminisation " en établissant un portrait statistique (nombre de réalisatrices, de programmes, d'émissions dont elles sont responsables). Pour cela, il faudra trouver les listes, fouiller les archives, les génériques, compter, enregistrer. Mais il peut aussi s'agir d'analyser la production féminine du métier. Déjà ici des précisions s'imposent au terme de production. Que faut-il entendre par "production ou pratique féminine dans la réalisation »? La réponse à la question peut se faire sur deux registres empiriques complètement distincts.

D'une part, cela signifie l'actualisation par des femmes de pratiques jusque-là articulées par des valeurs et des normes instaurées principalement par des hommes. En supposant que cela est réalisable sans trop de difficultés ${ }^{12}$, il faut mettre en route des protocoles d'observation et d'enregistrement. Les choix sont multiples et ne sont pas étrangers au budget défini, au temps disponible, au climat dans les équipes de travail. Recueillir des informations sur les pratiques [questionnaires, histoires de vie...] oriente la recherche dans les « discours sur » qui ne peuvent être que naïvement confondus avec les pratiques réelles. Observer en direct en suivant les négociations dans les choix thématiques et en travaillant sur les plateaux [observation dite participante] entraine les "chercheuses » sur le terrain glissant du double jeu d'observatrices et d'actrices censées ne pas prendre cause. Rédiger et passer des questionnaires, c'est non seulement mettre en place un protocole de recherche complètement différent de celui de l'observation participante, mais c'est aussi obtenir des données d'observation, des résultats complètement différents.

L'interprétation de ces données pose un autre ordre de questions. Va-t-on se contenter de commenter ces analyses descriptives ou faut-il montrer/évaluer la spécificité de la " féminisation des réalisations télévisuelles »? Répondre de la spécificité des situations observées exigera des observations comparées avec la féminisation d'autres métiers et professions. Ce faisant, les "chercheuses " s'imposent un protocole de décentration et une mise en perspective des observations dans le cadre d'une problématique plus générale. Cette décentration dépasse l'approche sociographique et situe la recherche dans le champ de la sociologie du travail et des professions.

D'autre part, cette "féminisation » peut être approchée par l'analyse des produits télévisuels. Il s'agirait alors de faire voir ce que des réalisatrices approchent et comment elles traitent, "produisent» des regards féminins sur. Colliger et analyser des productions télévisuelles demandent évidemment la mise en marche de protocoles bien différents de ceux qui portent sur l'enregistrement de pratiques professionnelles. Encore une fois, l'interprétation des observations devra opter pour la lecture comparative afin de 
montrer la différence ou la non-différence entre regards féminins et masculins malgré les contraintes organisationnelles et technologiques.

Enfin, la question de l'influence des réalisatrices sur les modèles culturels diffusés est un volet beaucoup plus complexe tant au plan théorique que pratique. Non seulement faut-il d'abord évaluer la spécificité des messages transmis par les réalisatrices (voir supra), mais aussi faut-il être en mesure d'évaluer leurs " effets » sur les valeurs et normes partagées par les téléspectateurs et téléspectatrices. Pouvoir faire la part des différents médias et de leur évolution dans les visions du monde d'une population n'est pas une mince opération. Le chemin d'une sociologie naïve consisterait à organiser un sondage statistiquement très représentatif et ainsi tout à fait scientifique pour demander aux gens quelle est « l'influence » que telle série télévisuelle a pu avoir sur leur vie.

\section{Structure idéologique d'une population}

On prétend décrire l' «idéologie politique » d'une population à partir d'une échelle d'attitudes construite avec seulement des items traduisant des orientations sur une dimension gauche/droite ou libéralisme/conservatisme. La manipulation technologique n'est pas défaillante, puisque la consistance interne de l'échelle obtenue dans l'échantillon est très élevée. Mais l'analyse critique révèle deux faiblesses méthodologiques de taille.

Premièrement, l'échelle mesure une dimension attitudinale. Une seule échelle d'attitudes n'opérationnalise pas le concept d'idéologie. Le calcul sur l'unidimensionnalité d'une échelle ne mesure pas la structure d'un système idéologique. Il confirme la fiabilité et la validité d'une mesure sans synthétiser une « structure idéologique ». Pour cela, il faudrait examiner les relations entre plusieurs croyances enregistrées à l'appui de plusieurs échelles. Donc faiblesse dans le jugement d'existence.

Deuxièmement, l'échelle est construite avec un échantillon sémantiquement réduit aux seuls items de l'ensemble gauche-droite ou libéral-conservateur. Cette donnée de sondage n'est pas une donnée sociologique rencontrée dans l'action sociale ou sur le marché de la communication publique, elle est le produit construit à partir du protocole qui ne mesure que ce qu'il enregistre. C'est en quelque sorte forcer les réponses à entrer dans la logique "gauche/droite» ou «libéral/conservateur» a l'exclusion de toute autre dimension idéologique.

Ces trois applications montrent que la méthode et ses protocoles sont liés à la genèse d'un projet de recherche et que celle-ci est un moment important déterminant la mise en chantier des étapes d'application empirique. On peut dire que la genèse institue son objet et que son traitement, c'est-à-dire la méthode appliquée tant dans les opérations théoriques que les manipulations empiriques, le constitue objet de science.

Les opérations théoriques définissent l'objet de la recherche et son contexte significatif. Elles construisent les structures significatives premières, c'est-à-dire celles qui servent de cadre de référence à l'intérieur duquel une explication est cherchée. Ici la méthodologie s'intéresse principalement à la problématique, au travail conceptuel et à la construction de l'objet par l'utilisation de schémas explicatifs, de paradigmes et de modèles. 


\section{Méthodes et techniques}

42 La mise au point du terme "méthode" qui vient d'être faite laisse entendre que les méthodes sont bien souvent des techniques d'application. Comme il a été dit à plusieurs reprises, il peut y avoir plusieurs chemins pour réaliser l'observation ou l'expérience dans l'empirisme. Le terme "méthodes" désigne ainsi un ensemble d'opérations qui permettent, primo, d'observer et d'enregistrer un corpus d'informations conventionnellement appelées des "données" et, secundo, de les traiter selon des protocoles très précis. Les techniques sont les opérations pour observer, décrire, classer, mesurer. Ce sont des opérations de manipulation, de traitement. Elles sont très souvent rendues possibles après une application numérique qui code un classement, un ordre, une métrique. La méthodologie s'intéresse avant tout à la logique opératoire, qui annonce la construction de l'objet, et non aux opérations elles-mêmes.

Les techniques correspondent aux règles de conduite à suivre après certains jugements de méthode. Ces dernières sont sous une juridiction autonome que nous pouvons appeler la technologie scientifique. Il s'agit de produire des données pour ensuite les traiter en appliquant un savoir-faire qui respecte les propriétés formelles inhérentes à chaque technique. Ceci requiert une compétence pratique parce que, bien souvent, il s'agit d'adapter des connaissances ou des expériences pratiques développées dans une autre discipline ${ }^{13}$. Les manipulations empiriques appartiennent aux domaines de la traduction concrète des concepts et de la preuve. Cette distinction est fondamentale parce que la technique la plus avancée peut être basée sur des erreurs méthodologiques irréversibles qui disqualifient la validité de la recherche.

44 La confusion entre méthodes et techniques est malheureusement très répandue. Un flou se rencontre même dans des ouvrages de méthodologie qui définissent leurs concepts à partir du langage ordinaire. Ainsi, P. Massé, présente les méthodes par rapport aux techniques comme des démarches non codifiées ni formalisées dans le détail, les techniques l'étant (1992, p. 23).

Quand les médias parlent de la méthodologie du sondage, ils tiennent à justifier sa scientificité en exposant rapidement le mode de cueillette des informations, les taux d'échantillonnage et de réponse. Mais ils gardent bien un silence sur les décisions concernant la rédaction des questions, leur ordre dans le questionnaire, l'éventail serré des réponses, la signification du taux des indécis... La méthode est réduite ainsi au domaine apparemment ardu et solide de la statistique et du calcul : elle vient garantir le sérieux du sondage, elle le rend "scientifique»! En fait, les maisons de sondage appliquent un ensemble de techniques de questionnaire et d'échantillonnage avec des « professionnels » experts en questionnement téléphonique comme si la validité de cette technique de cueillette va de soi. Le savoir-faire technique occulte l'examen méthodologique sur la possibilité même d'enregistrer les opinions souhaitées et d'évaluer la compétence des répondants.

Pour sortir du labyrinthe conceptuel, faisons le point en établissant un premier schéma.

Notre lecture des concepts utilisés nous permet de travailler avec deux axes principaux. Le premier axe détermine les étapes allant de l'abstraction/réflexion jusqu'aux réalités empiriques. L'abstraction suprême serait alors la réflexion sur les conditions mêmes de la production d'idées et de cognition. C'est la sphère de l'épistémologie et de la méthodologie au sens fort du terme. À l'extrême empirique, nous retrouvons les 
techniques. Cet axe passe de l'ordre du possible à celui du comment. Le deuxième axe oppose le descriptif au normatif. Le schéma croisant les deux axes permet de situer l'espace conceptuel occupé par les termes rencontrés jusqu'à présent. On peut voir que la réflexion méthodologique peut être normative ou positive. L'épistémologie occupe généralement un espace normatif, mais elle peut être critique, voire positive. La figure I montre les différentes sémantiques du terme « méthode ».

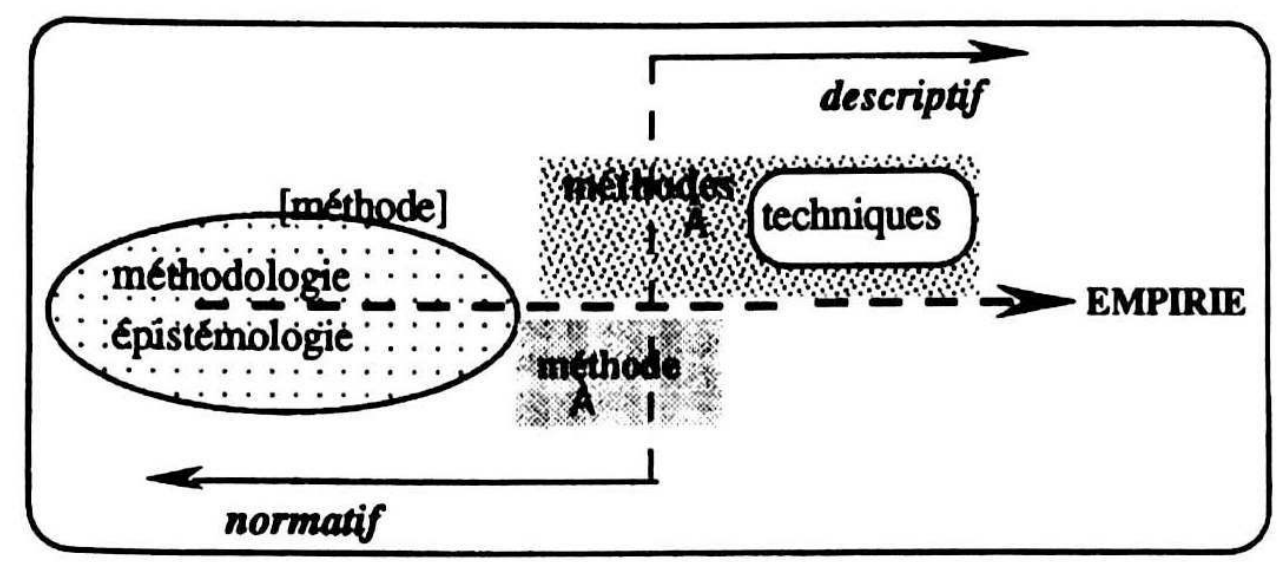

Figure 1. Schéma conceptuel des ouvrages qui lui sont consacrés, suit l'approche d'une epistémologie cartésienne à dose fortement normative. Les ouvrages commencent invariablement par définir la méthode scientifique et ses corrélats habituels comme l'objectivité, la preuve. Ils posent la raison expérimentale comme postulat de vérité. C'est pourquoi ils sont en quelque sorte programmés, d'une part, pour fournir un lexique méthodologique et technique et, d'autre part, pour initier aux techniques de la science normale. Rivés aux techniques de 
recherche introduites par un rappel rapide des conditions élémentaires du savoir scientifique, beaucoup d'ouvrages de méthodologie en sciences humaines sont des manuels de méthodologie normative. C'est pourquoi un grand nombre de manuels ${ }^{14}$, optant implicitement pour une définition normative de la science, sont essentiellement orientés vers la construction de variables et les calculs sur des «données » sans porter l'attention sur la genèse du problème posé ni sur les choix subtils qui président à la définition de l'objet de recherche. Ils ne font pas entendre qu'il peut toujours ne s'agir que d'unedéfinition, d'une problématique parmi tant d'autres.

Ceci laisse penser que la méthode a déjà acquis toutes les garanties de sa scientificité dans ce domaine comme dans tous les autres des sciences humaines. Cette façon d'ouvrir le chemin vers la production scientifique s'inscrit ainsi nécessairement dans une logique normative. Même si cette démarche reste critique et ne se limite pas à la simple dictée des règles à suivre, elle répond toutefois directement à une préoccupation normative en visant l'objectivité et la vérité, posées comme produits immédiatement accessibles. Ce faisant, ce type de démarche fait l'économie d'une réflexion sur l'outil premier de la production scientifique constitué par son langage, voire tout langage. On pourrait dire qu'une telle méthodologie indique tout bonnement le parcours à suivre sans attirer l'attention sur ce qui permet de faire un chemin.

Ces ouvrages sur les méthodes consacrent peu de place, voire aucune, à une réflexion systématique sur le langage, ils soulignent inévitablement l'importance des concepts théoriques et consacrent une bonne partie de leur démarche à leur opérationnalisation, comme point de départ de la recherche scientifique. La réflexion méthodologique porte alors seulement l'attention sur les opérations manipulatrices nécessaires au passage du théorique qui travaille sur un abstrait à l'empirique qui opère sur un concret. Tout porte à croire, dans ces manuels, que la production théorique semble être dispensée de toute méthode et que ses conditions de possibilité échappent à une méthodologie. C'est oublier que le langage scientifique hérite lui aussi de la crise due au repositionnement du langage dans le champ de la connaissance.

Le statut critique et réflexif que devrait prendre une méthodologie positive fait aussi appel à un dialogue plus nourri entre les théoriciens et les empiristes. Dans un certain sens, par une réflexion plus serrée sur la production théorique même et sur le passage entre les langages théoriques et les langages observationnels, l'attention méthodologique annule la dichotomie traditionnelle entre la théorie et la recherche empirique. En effet, une telle attention ne peut que développer une théorie mettant en "parallèle " l'opérationnalisation des catégories et les schémas théoriques. Cette exigence laisse voir la nécessité d'élaborer des théories "auxiliaires» (Blalock, 1968, pp. 23-26) censées incorporer dans la réflexion dite théorique d'importantes décisions ou définitions des situations concrètes servant à mettre une théorie à l'épreuve des faits. Dans cette perspective, la méthode rendra un grand service à son statut épistémologique. En articulant de façon plus explicite le passage du théorique à l'empirique, la méthode ne peut plus ignorer la position inconfortable qu'elle occupe en sémantisant des termes théoriques par un retour dans l'empirique, ce retour demeurant toujours une interprétation que tout calcul ou toute manipulation technique ne peuvent occulter. Dans ce sens, tout retour à la méthode devient un détour théorique. 


\section{BIBLIOGRAPHIE}

ABU-LABAN S., ABU-LABAN B., « Women and the aged as minority groups. À critique. » in Marshall, V.W. (éd.), Aging in Canada, Toronto, Fitzhenry \& Whiteside, 1980, pp. 63-79.

BERG D.N., SMITH K.K., Exploring clinical methods for social research, Beverly Hills, Sage, 1985.

BLALOCK H. M., « The measurement problem : a gap between the language of theory and research », in BLALOCK H.M., BLALOCK A. B., (éd.) Methodology in social research, New York, McGraw-Hill, 1968, pp. 5-27.

CHARON J., LEMIEUX J., SAUVAGEAU F., Les journalistes, les médias et leurs sources, Montréal, G. Morin, 1991.

DURAN J., Toward a feminist epistemology. Savage, MY., Rowman \& Littlefield Pub, 1991.

EMMERT Ph., BROOKS W.D., Methods of research in communication, Boston, Houghton Mifflin Co., 1970.

FEYERABEND P., Contre la méthode. Esquisse d'une théorie anarchiste de la connaissance. Paris, Le Seuil, 1988.

FREY R. et alii, Investigating communication : an introduction to research methods, Englewood Cliffs, N.J., Prentice Hall, 1991.

GARFINKEL H, « The origines of the term « ethnomethodology » », in Turner, R. (éd.), Ethnomethodology, Harmondsworth, Penguins Books, 1975, pp. 15-18.

HSIA H.-J., Mass communication research methods : a step-by-step approach, Hillsdale N.J., Erlbaum Associates, 1987.

KUHN T., La structure des révolutions scientifiques, Paris, Flammarion, 1972.

LARAME A., La recherche en communication. Éléments de méthodologie, Sillery, Presses de l'Université du Québec, 1991.

LEBEL E., Lavallée, M., « Création, maîtrise technique et gestion : les réalisatrices à la télévision québécoise », Recherches Féministes, vol. 9,1996, pp. 57-80.

LEDRUT R., « Méthode ou méthodes? », Cahiers internationaux de sociologie, LXXXI, 1987, pp. 215-225.

MASSE P., Méthodes de collecte et d'analyse de données en communication, Sillery, Presse de l’Université du Québec, 1992.

MCPHERSON B.D., Aging as a social process, Toronto, Butter-worths, 1990.

MORIN E., La méthode. I La nature de la nature, Paris, Le Seuil, 1977.

NAMENWIRTH J.Z., MILLER R.L., WEBER R.P, « Organizations have opinions : a redefinition of publics », Public Opinion Quaterly, 45,1981, pp. 463-476.

PEACE S., "The forgotten female : social policy and older women », dans Phillipson, C, A. Walker, Ageing and social policy, a critical assessment, Aldershot, Gower, 1986, pp. 61-86.

PIAGET J., Épistémologie des sciences de l'homme, Paris, Gallimard, 1972. 
REINHARZ S., Feminist methods in social research, New York, Oxford University Press, 1992.

SCRIMGER J., « Profile : women in Canadian public relations », Public Relations Review, XI/3,1985, pp. 40-46.

STEMPEL G.H., WESTLEY, B.H., Research methods in mass communication, Englewood Clifs N.J., Prentice-Hall, 1989.

TARDY Ch. H., À handbook for the study of human communication, Norwood NJ, Ablex Publishing Corp., 1988.

ULLMO J., La pensée scientifique moderne, Paris, Flammarion, 1969.

WARD J., HANSEN K.A., Search strategies in mass communication, New York, Longman, 1987.

WIMMER, R.D., DOMINICK, J.R., Mass media research : an introduction, Belmont Ca., Wadsworth, 1991

\section{NOTES}

1. La meilleure preuve a l'appui est certainement le critère de représentativité d'un échantillon. Cette seule caractéristique confirmée laisse parler d'un échantillon scientifique. Mais n'est-ce pas consacrer une science du normal, du commun en faisant place a l'aléatoire au mépris du caché (G. Bachelard) et de l'atypique hautement informant?

2. Certains préféreraient lire ici le terme vérité en tant que toute adéquation empirique apporte une certitude.

3. Ceci soulevé le sujet complexe des rapports entre expérience empirique, abstraction et pensée déductive, que la méthode expérimentale considère comme allant de soi

4. Les instances universitaires ou gouvernementales des subventions de recherche ne le cachent pas par les étiquettes données à des recherches dites «stratégiques ", la formation d' "équipes d'émergence »...

5. Voir Scrimger J. (1985).

6. McPherson remarque le paradoxe (1990, p. 141). Abu-Laban, Abu-Laban(1980) discutent des implications théoriques de l'application du concept de minorité Peace (1986) parle de la femme âgée en tant que « femme oubliée ».

7. Laissons-nous supposer que la connaissance objective du marché du travail a préalablement défini cette situation. $\mathrm{D}$ faut bien admettre qu'une définition réductrice consisterait i prétendre qu'il y a peu de femmes travaillant en relations publiques et que, par conséquent, tout échantillon féminin ne serait pas « représentatif» de la population.

8. On peut comprendre qu'un tel discours réducteur soit qualifié de «patriarcal » par des chercheuses féministes.

9. Garfinkel (1975) raconte qu'il construisit le néologisme d'epistemology comme la connaissance des régies de procédures profanes des gens ordinaires par analogie à l'ethnomédecine, l'ethnobotanique qui qualifient la connaissance respective de la médecine ou de la botanique d'un groupe.

10. Voir les méthodes des travaux de Sauvageau, Lemieux, Charon (1991) et Namenwirth, Miller, Weber, (1981).

11. Voir Lebel, Lavallée (1996) pour un traitement empirique de la problématique ici soulevée.

12. Ceci semble s'avérer beaucoup plus délicat quand on sait que les réalisations deviennent de plus en plus des productions d'équipes mixtes. 
13. À titre d exemple, le questionnaire et l'enquête ont d'abord été des opérations policières, judiciaires et administratives. La psychothérapie et la psychanalyse ont instauré l'entretien non directif. Les échelles d'attitude sont des mises au point de la psychophysique.

14. Voir Emmert, Brooks (1988), Frey, Al. (1991), Hsia (1987), Laramé (1991), Masse (1992), Stempel, Westley (1989), Tardy (1988), Wimmer, Domini ck (1991).

\section{RÉSUMÉS}

Le concept de méthode couvre deux champs distincts. Il a une portée descriptive dans la présentation des étapes d'une recherche et une portée normative par son renvoi à un ensemble de règles de scientificité. La méthodologie, en tant que réflexion sur ces deux modes, peut analyser les pratiques suivies dans une recherche ou prescrire des règles à appliquer. Une tradition tente à confondre méthodes et techniques. Cette restriction méthodologique repose sur la définition dominante de la connaissance expérimentale. Ceci est particulièrement observé dans les manuels de méthodologie sur la recherche en communication.

The concept of method covers two very different fields. It has a descriptive meaning in the presentation of the steps followed in research and a normative one when it refers to a set of rules. Methodology, as a reflexion upon these two meanings, may analyse the practices of a research project or prescribe rules to be applied. À scientific tradition tends to confound methods and technics. Such a methodological restriction is based upon a prevalent definition of experimental knowledge. This seems particularly evident in textbooks of methodology in communication research.

INDEX

Mots-clés : méthode, méthodologie, norme, protocole, science, techniques

\section{AUTEUR}

\section{PAUL STRYCKMAN}

Professeur à l'université Laval où il enseigne les méthodologies de recherche. Sociologue, il fait notamment autorité en sociologie des religions. Il a mené des travaux importants aux États-Unis et au Mexique et est régulièrement professeur-invité aux universités de Bordeaux et de Louvain-la-Neuve. 